\title{
Atmospheric dust as a possible survival factor for bioaerosols
}

\author{
Jun Noda ${ }^{1, *}$, Sota Tomizawa ${ }^{1}$, Buho Hoshino ${ }^{1}$,Erdenebadrakh Munkhjargal ${ }^{2}$, Kei Kawai ${ }^{3}$, and Kenji Kai $^{4}$ \\ ${ }^{1}$ Rakuno Gakuen University, Ebetsu 069-8501 Hokkaido, Japan \\ ${ }^{2}$ National Agency for Meteorology and Environment Mongolia, Ulaanbaatar, Mongolia \\ ${ }^{3}$ Nagoya University, Chikusa-ku Nagoya 464-8601 Aichi, Japan \\ ${ }^{4}$ Ibaraki University, Bunkyo Mito 310-8512 Ibaraki, Japan
}

\begin{abstract}
In this study, we present some of the laboratory measurements of the viability of bioaerosols together with different environmental dust to understand the interaction among those components. Model airborne bacteria, DH5 $\alpha$ Escherichia coli, was used to assess the dust affecting a viability reduction rate of the DH5 $\alpha$ bacteria in a Teflon reaction chamber. The viability reduction rate of the DH5 $\alpha$ model airborne bacteria was measured with a culture method. The DH5 $\alpha$ bacteria were nebulized into the chamber and airborne dust materials 1) Phosphate Buffer Solution (PBS) as a control, 2) desert sand from Mongolia and 3 ) sludge dust from the coastal area of Japan. The result indicated that the co-existence of DH5 $\alpha$ with desert dust from Mongolia significantly decreased the viability and with the sludge dust from Japan significantly increased the viability of the airborne DH5 $\alpha$ compare to the control PBS dust $(p<0.05)$. Furthermore, soot as a model air pollutant was generated by a candle and mixed with a model airborne bacteria Mycobacterium smegmatis. The results indicated that the different types of airborne environmental dust influenced the viability of airborne bacteria with the DH5 $\alpha$ experiments. Furthermore, the presence of soot indicated a possible protective effect of increasing the survival rate of Mycobacterium smegmatis.
\end{abstract}

\section{Introduction}

Desert regions continue to discharge a large amount of, and they circulate around the globe [1]. These desert dust bring the important minerals to a distant area and supply essential elements as nutrients for various trophic levels. In other cases, the dust from the Saharan desert was suspected to act as the carrier for biological components such as fungus across the Atlantic Sea to the Caribbean sea to cause coral leaf damage [2]. Furthermore, Iwasaka et al.[3] reported the presence of nucleic acid on the surface of the dust in China, indicated an abundance of some biological components as a part of the dust from the desert atmosphere. The aerosols with biological components as bioaerosols can be transported a long distance which may reach downwind and/or surrounding regions to cause possible health effects to human and livestock.

For example in human, the number of patients with tuberculosis has been decreasing; however, the number of patients diagnosed with Non-tuberculous mycobacteriosis (NTM) tend to be increasing mainly in middle-aged women in Japan. There are also some regional differences with a type of bacteria, which may be caused by the environmental factor associated with atmospheric dust and pollutants. Since the NTM cases are almost always found in the respiratory system, an infection via airborne pathway is the most plausible one to spread among the human population and possibly livestock animals. Also, in the highly polluted industrial region, the number of patients infected by
Mycobacterium kansasii tends to be higher [4, 5]. Although the detailed mechanisms are not fully understood yet, environmental dust including pollutants may play an important role to determine the survival of bioaerosols. In this study, we try to understand how the co-existence of different dust or pollutants may act the survival of bioaerosols in laboratory studies.

\section{Material and Method}

\subsection{Chamber system}

For the examination of the survival of bacterial bioaerosols, two chamber systems were employed. Figure 1 indicates a $128 \mathrm{~L}$ Teflon chamber system, which has an internal separator to divide two equal size compartments for the preparation of two different aerosolized materials before mixing them together.

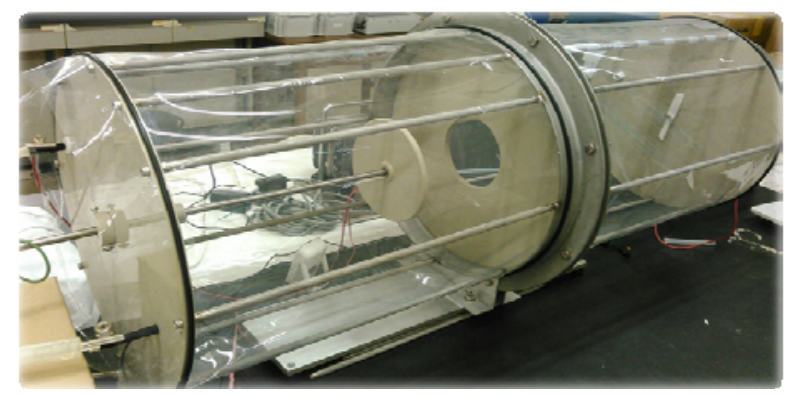

Figure 1. The Teflon chamber system with $128 \mathrm{~L}(64 \mathrm{~L} \times 2$ compartments).

\footnotetext{
* Corresponding author: jnoda@rakuno.ac.jp
} 


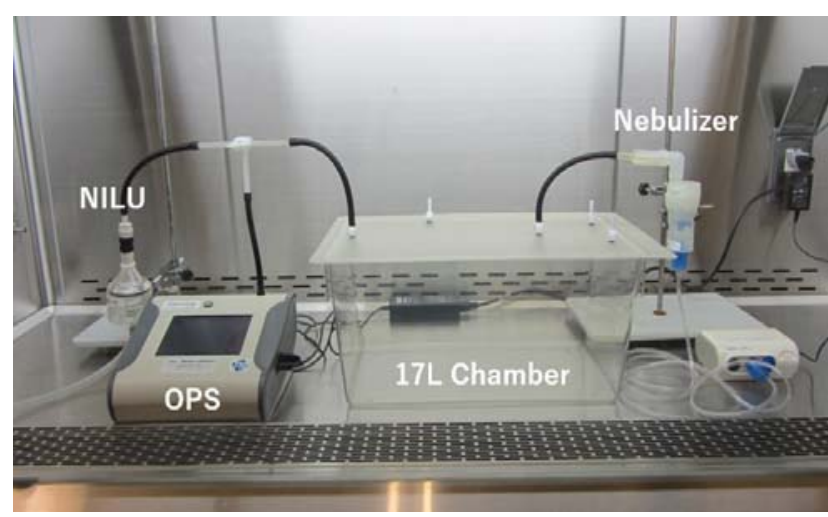

Figure 2. The polycarbonate chamber system with $17 \mathrm{~L}$. Nebulizer OPS and NILU filter unit are attached to the chamber.

Figure 2 indicates a $17 \mathrm{~L}$ polycarbonate chamber with polypropylene lid without the internal separator; also some other instrumentations are reported herein.

\subsection{Model Bioaerosols}

The E. coli (DH5 $\alpha$ ) was used as model bacteria to simulate the survival of bioaerosol under the different conditions. E. coli solution was prepared in phosphate buffer solution (PBS), and E. coli was $10^{8}$ to $10^{9}$ colony forming unit (CFU) per $\mathrm{ml}$. For the experiment with Mycobacterium smegmatis $10^{7}$ to $10^{8} \mathrm{CFU}$ was used together with PBS and bovine serum albumin (BSA). The $128 \mathrm{~L}$ chamber was used for the E. coli bioaerosols experiments, and $17 \mathrm{~L}$ chamber was used for $M$. smegmatis experiments.

\subsection{Aerosol generation}

For the generation of dust aerosols, the dust aerosol generator was prepared with of conventional impinger and silicon tube as shown in Figure 3. The particle free air was introduced through silicon tube to blow off and emitted out dust particles. Emissions of coarse particles were limited by glass filter inside the impinger. For some experiment, soot was generated as Figure 2 indicates a compressor type nebulizer (NE-C30, Omron, Japan) which was employed to generate aerosolized bacteria.

\subsection{Particle counting}

Particle measurements were carried out with an optical particle counter (OPC: AeroTrak Model 9306, TSI, USA) and an optical particle sizer (OPS: Model 3330 TSI, USA). Both OPC and OPS were employed to count the number of the bioaerosols and other particles. Before all the experiments, chamber systems were purged with a particle-free air with High-Efficiency Particulate Air (HEPA) filter unit. After the purge process, inside the chamber system were monitored with OPC or OPS to confirm particle free conditions.
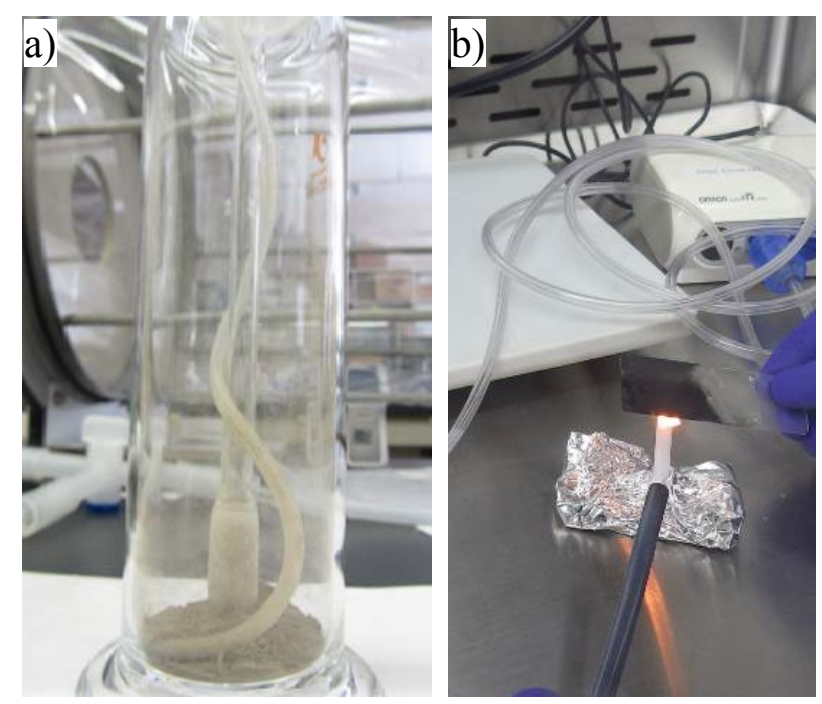

Figure 3. a) The dust aerosol generator with silicon tube as air intake to blow air and the middle glass filter to select out large size particles (left). b) The soot generation with incomplete combustion of candle (right).

\subsection{Preparation of aerosols in the chamber}

For the experiment with the $128 \mathrm{~L}$ chamber, one compartment was filled with dust, and another compartment was filled with bioaerosols. After the filling process the aerosols were stabilized for ca. $5 \mathrm{~min}$. Then particle numbers were measured to confirm equivalent concentrations of bioaerosol and dust. Then, the middle separator was open to let two components to interact together in one compartment for ca. $60 \mathrm{~min}$.

\subsection{Aerosol sampling from the chambers}

For the collection of aerosols, Bio-sampler or NILU filter unit was used. Bio-sampler is similar to impinger which collects aerosols into a liquid with pumping out air through it for few minutes. Bio-sampler has three angled nozzles, causing impingement of aerosols in vortex motions to increase the collection efficiency. After the sampling, the liquid solution was transferred to a sterile test tube and centrifuged for few minutes to concentrate sampled bacteria to the bottom section of the test tube and the supernatant was discarded. Only the bottom solution was transferred to an agar plate with different dilution steps for CFU determination.

\subsection{Dust properties}

In this work, Gobi desert dust and Ishinomaki sludge dust were examined by the scanning electron microscope (SEM). Also, the chemical properties of the dust were examined by loss on ignition and X-ray fluorescence spectrometer.

\subsection{Statistical analysis}

Student's $t$-test was used to determine statistically significant differences. A $P$ value of $<0.05$ was considered to be statistically significant. 


\section{Result and discussion}

\subsection{Dust property}

For the experiment with $E$. coli (DH5 $\alpha$ ) viability test, the effect of Gobi desert dust and Ishinomaki coastal sludge were different dust types were examined which indicate in Figure 4 a) and b), respectively. The Gobi dust image, a) has a surface structure with a scale like a shape that smaller fraction may come off freely and emit as fine particulate matters. The Ishinomaki sludge dust image, b) indicate somewhat a spiky form with a rough surface area than Gobi dust, it has more aggregation of substances which may come from the marine sediment. Since the size of those particles is too large to be suspended as whole particles in the chamber systems or the real atmospheric environment. Thus the fraction of these particles is considered to be important for the survival of bioaerosols.

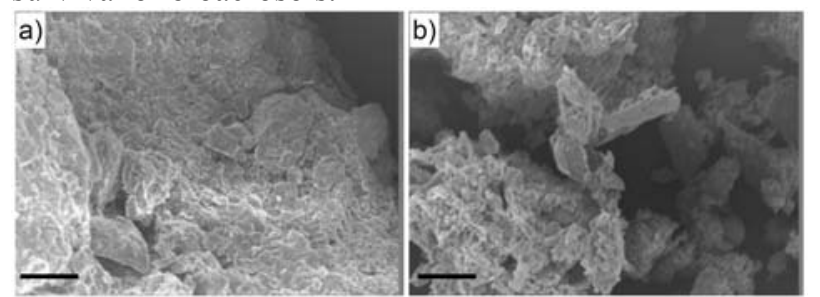

Figure 4. The SEM image of a) Gobi desert dust (left) and b) Ishinomaki sludge dust (right). Scale bars $=10 \mu \mathrm{m}$.

Furthermore, chemical analysis of Gobi and Ishinomaki dust indicated an organic fraction, calcium, and iron concentrations were greater with Ishinomaki dust. The ignition loss result indicated $1.2 \%$ and $5.6 \%$ organic matter from Gobi desert dust and Ishinomaki sludge dust, respectively. The organic matter of the Ishinomaki dust showed about 4.7 times higher value than the Gobi dust. Similarly, the calcium and iron concentrations of Ishinomaki sludge dust were 3 and 4 times higher, respectively.

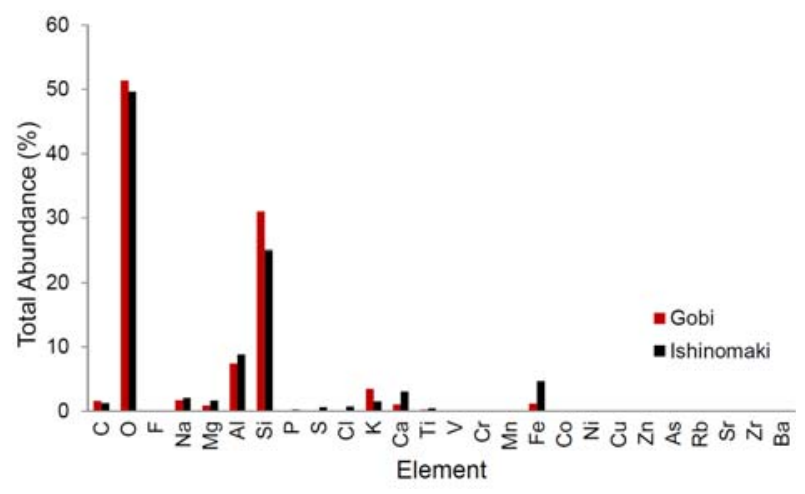

Figure 5. The X-ray fluorescence spectrometer analysis result of Gobi desert dust and Ishinomaki sludge dust indicated for 26 elements.

For the particle size information of dust, Figure 6 indicates the field measurement in Gobi desert area with thinner lines, DZ0429, TO0430, and DZ0430. The Ishinomaki sludge and Gobi desert dust are indicated with a thicker line. The data from the field measurements indicated a slightly different size distribution pattern from laboratory measurements. The field data sets show

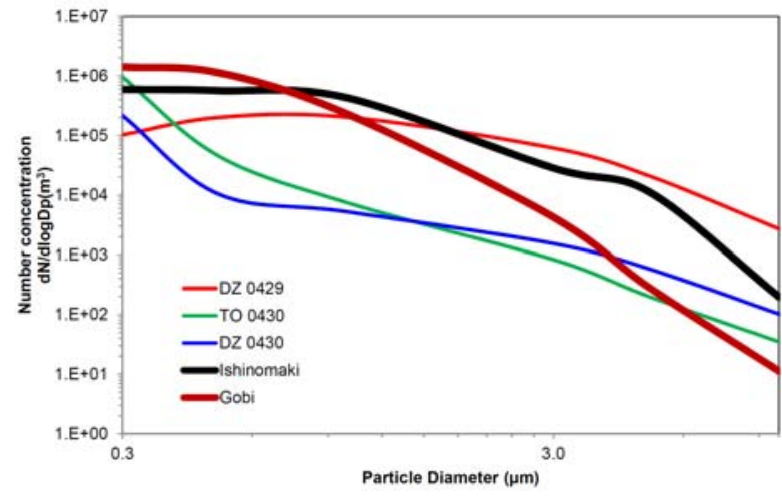

Figure 6. The dust size distribution measurement by OPC from Gobi field measurements (DZ0429, TO0430, and DZ0430) and laboratory generated Ishinomaki sludge and Gobi desert dust.

lower concentrations of dust particles from 0.5 to about $3 \mu \mathrm{m}$. For the $0.3 \mu \mathrm{m}$ particle size, the laboratory measurements and TO0430 were about the similar range, which indicates the laboratory generated dust were comparable to the real field situation. Since the DZ0429 was the dust storm event condition, the laboratory measurement was similar to the situation in the field. However, 0.3 and $10 \mu \mathrm{m}$ size range indicated less concentration and higher concentrations, respectively. Since the DZ0429 was the measurement of dust storm event, a high concentration of larger sizes particles (above $3 \mu \mathrm{m}$ ) was observed. Possibly, the presence of the large size dust particles may hinder the laser light to reach the small size fraction of the particles to cause less scattering.

\subsection{Dust affecting viability of bioaerosols}

These dust samples were further examined for the viability of bioaerosols in the chamber system. The reduction of viability was calculated by comparing initial bacterial CFUs on the nebulizer (Neb.) liquid and Biosampler (BS) liquid, as indicated on the calculation formula (1).

$$
\text { Reduction rate }=B S C F U / N e b . C F U
$$

The result of different dust samples and bacteria are indicated in Figure 7. The PBS driven particle as control dust and comparison was made with Gobi and Ishinomaki dust samples. The viability reduction rate of the DH5 $\alpha$ bacterial CFU was measured with the culture method. The DH5 $\alpha$ bacteria were nebulized into the chamber, and airborne dust materials were mixed in the chamber. The phosphate Buffer Solution (PBS) as control dust indicated the $10^{-5}$ order of reduction and, desert sand from Mongolia, and sludge dust from the coastal area of Japan. The result showed that the coexistence of DH5 $\alpha$ with desert dust from Mongolia had significantly higher reduction rate and the Ishinomaki sludge dust had significantly lower reduction rate $(p<0.05)$. From this experiment, the presence of Ishinomaki dust has attributed to lower the reduction rate by exposing bacteria inside the chamber system. A possible reason for this difference might be connected with the physical and chemical properties. For the physical property differences, as indicated on the Fig. 4, 
Ishinomaki dust has more spiky surface shape than Gobi desert dust. Such surface shape with a large roughness may have acted as a shelter for the aerosolized E. coli from the drying process. For the chemical property differences, the 4.7 times higher organic matter was observed with the Ishinomaki dust, which may have a higher affinity with bacterial phospholipids found on the E. coli cell membrane. Furthermore, the E. coli cell membrane contains lipopolysaccharides, which are known to have substantial negative charges. Since $\mathrm{Ca}$ and $\mathrm{Fe}$ concentrations were 3 to 4 times higher in Ishinomaki dust than Gobi dust, they may have positively charged form as $\mathrm{Ca}$ and $\mathrm{Fe}$ cations to have a higher affinity with $E$. coli. The affinities between the bacteria and dust may result in the formation of the wellattached bacteria-dust component, which may prevent from dryness in the atmosphere to extend the viability of bacteria as bioaerosols. As a limitation, this study alone cannot verify the attachment mechanism of bacteria and dust in the airborne form.

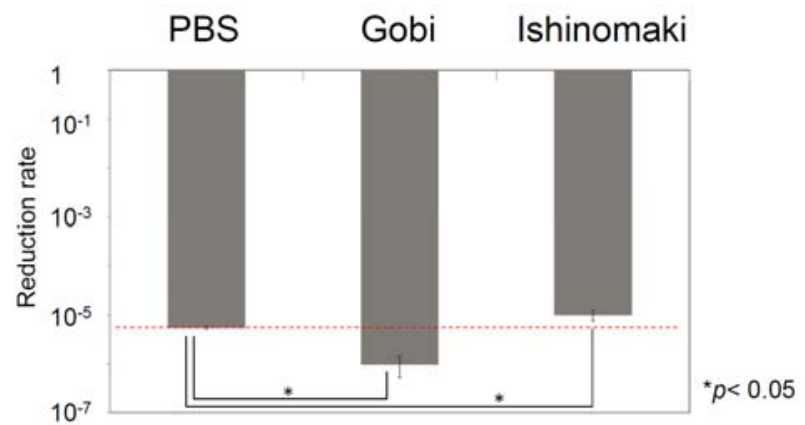

Figure 7. The results of viability tests with PBS as control, Gobi desert dust, and Ishinomaki sludge dust.

\subsection{Soot affecting the viability of bioaerosols}

Since the higher organic content may attribute to prolong the survival of bacteria, soot was considered as one component to be examined for the effect of aerosolized bacteria experimentally. For this experiment, Mycobacterium smegmatis (M. smeg) was used as a model bacteria because of similar characteristic to NTM, yet much lower pathogenicity. Since the Mycobacterium can survive in the soil and aquatic environment, they are rather persistent and difficult to assess the change of the viability. Thus the viability change was examined with UV exposure as stress. Although, the E. coli experiment could not verify the attachment of bacteria and dust in the air; this experiment examined bacteria and soot aerosols collected on the filter surface as a co-existing site to investigate which effect can be observed for bacteria and soot resided tightly together. The result indicated that there is no significant effect for bacteria growth with the presence of the soot together. Since soot is considered as air pollutants and which is known to contain some carcinogens such as polyaromatic hydrocarbon (PAH). Although, the amount of PAH and other pollutants in the soot from the paraffin type candles can vary widely [6]. The amount of PAH and other organic compounds were not determined in this study, but it as model soot by making incomplete combustion in the laboratory. Figure 8 indicates the size distribution pattern of generated soot particles and
M. smeg bioaerosols. The soot particles were tended to have the high concentration at $0.3 \mu \mathrm{m}$, and it was expected to have even higher number concentration at the even nano-size range. Since the OPC has a limitation to measure down to $0.3 \mu \mathrm{m}$, there was no data available. The Bacteria particle size was tended to be higher than soot with a peak concentration of around $3 \mu \mathrm{m}$.

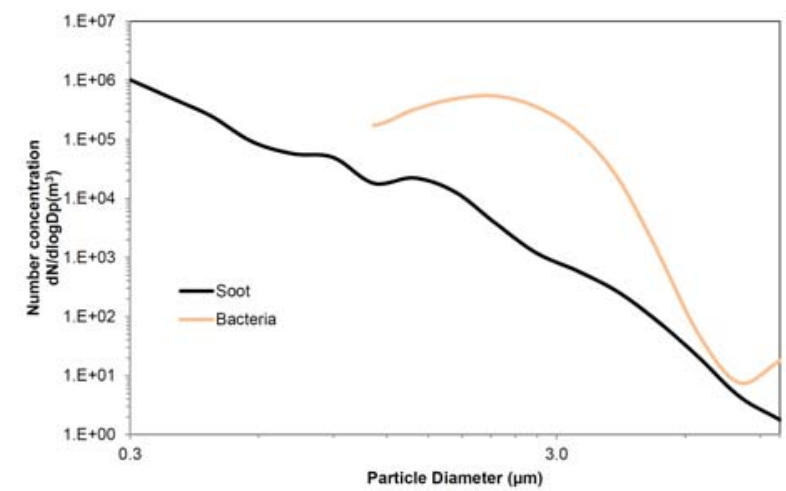

Figure 8. The dust size distribution measurement by OPC from laboratory-generated Soot and Bacteria particles.

For the result of UV exposure test, no significant differences were observed; however, with soot treated filter showed less reduction. Therefore, the protective function with soot for the airborne bacteria or other bioaerosols is a possible scenario to have pathogenic components to be transported a long distance and cause a health effect.

This work was supported by kaken $15 \mathrm{~K} 00568$ and JSPS Coreto-Core Program, B. Asia-Africa Science Platforms.

\section{References}

1. I. Uno, et al., Asian dust transported one full circuit around the globe, Nature Geoscience, 2 (8), 55, (2009)

2. V. Garrison, et al., African and Asian dust: from desert soils to coral Reefs, BioScience, 53 (5), 469480, (2003)

3. Y. Iwasaka, et al., Mixture of Kosa (Asian dust) and bioaerosols detected in the atmosphere over the Kosa particles source regions with balloon-borne measurements: possibility of long-range transport, Air Quality, Atmosphere \& Health, 2, (1), 29-38, (2009)

4. K. Mimura, The Trend of M. Kansasii infection in Okayama prefecture between 1994 and 2000, Kekkaku, 77 (10), 665-669, (2002)

5. S. Yoshida, et al., Present status of studies on epidemiology of Mycobacterium kansasii, in special reference to its epdemiology, Kekkaku, 86 (5), 515521, (2011)

6. M. Derudi, et al., Emission of air pollutants from burning candles with different composition in indoor environments, Environ Sci Pollut Res Int., 21 (6), 4320-4330, (2014) 